\title{
MicroRNA-489-3p plays a significant role in congenital hypothyroidism through regulating neuronal cell apoptosis via targeting translationally controlled tumor protein 1
}

\author{
QIN LIU*, YUEHONG LI* and YONG ZHOU \\ Department of Pediatrics, Yancheng Maternal and Child Health Hospital, Yancheng, Jiangsu 224002, P.R. China
}

Received June 22, 2020; Accepted November 24, 2020

DOI: $10.3892 /$ etm.2021.9660

\begin{abstract}
Accumulating reports have indicated that congenital hypothyroidism $(\mathrm{CH})$ is an endocrine disorder caused by underdeveloped thyroid gland or thyroid dyshormonogenesis. It has been also reported that certain microRNAs (miRNAs) may exert protective effects against the development of $\mathrm{CH}$. However, whether miR-489-3p regulates $\mathrm{CH}$ progression remains unclear. The aim of the present study was to investigate the effects of miR-489-3p on $\mathrm{CH}$ and elucidate the underlying mechanisms. Therefore, Sprague Dawley rats were injected with propylthiouracil (50 mg/day) to establish a $\mathrm{CH}$ model. Reverse transcription-quantitative PCR (RT-qPCR) assay demonstrated that miR-489-3p was upregulated in the hippocampal tissues of $\mathrm{CH}$ rats. Furthermore, the TargetScan software was employed to predict the target gene of miR-489-3p, and a dual luciferase reporter assay revealed that translationally controlled tumor protein 1 (TPT1) was directly targeted by miR-489-3p. Additionally, RT-qPCR and western blot assays suggested that TPT1 was markedly downregulated in the hippocampal tissues of $\mathrm{CH}$ rats compared with control rats. In addition, inhibitor control, miR-489-3p inhibitor, control-shRNA or TPT1-shRNA were injected into $\mathrm{CH}$ rats. The results of the open-field and forced swimming tests revealed that miR-489-3p inhibitor notably improved the behavior of $\mathrm{CH}$ rats. Flow cytometry was applied to explore the effects of miR-489-3p inhibitor on neuronal cell apoptosis, and the findings indicated that miR-489-3p inhibitor attenuated $\mathrm{CH}$-induced neuronal cell apoptosis, whereas these effects were reversed by treatment with miR-489-3p inhibitor and TPT1-shRNA. Finally, the function of miR-489-3p in neuronal cells was investigated in vitro. Neuronal cell viability,
\end{abstract}

Correspondence to: Professor Yong Zhou, Department of Pediatrics, Yancheng Maternal and Child Health Hospital, 31 East Century Avenue, Tinghu, Yancheng, Jiangsu 224002, P.R. China

E-mail: feichangren2006@126.com

*Contributed equally

Key words: microRNA-489-3p, neuronal cells, congenital hypothyroidism, translationally controlled tumor protein 1 apoptosis and the expression of apoptosis-related proteins were determined using MTT assay, flow cytometry and western blot analysis, respectively. The results demonstrated that miR-489-3p inhibitor enhanced cell viability, suppressed apoptosis and upregulated Pim-3, phosphorylated (p)-Bad (Ser112) and Bcl-xL expression. Rescue experiments indicated that these effects were reversed following silencing of TPT1. Taken together, the findings of the present study demonstrated that miR-489-3p inhibitor could relieve $\mathrm{CH}$-induced neurological damage through regulating TPT1 expression.

\section{Introduction}

Previous reports have revealed that thyroid hormone deficiency may lead to functional injuries, including congenital hypothyroidism $(\mathrm{CH})(1,2)$. Several developmental processes may be induced by $\mathrm{CH}$, such as disrupted neurogenesis and abnormal hippocampal neuron apoptosis (3-5). It was previously demonstrated that the majority of newborn infants with $\mathrm{CH}$ did not have significant clinical manifestations (6). It is widely accepted that the hippocampus is involved in cognitive activities in humans. Accumulating evidence has suggested that hypothyroidism promotes hippocampal neuron apoptosis (4,7-9). Moreover, a previous study suggested that perinatal hypothyroidism affects behavioral development and may lead to a decrease in spatial learning ability and memory, and these changes are closely associated with the increasing number of apoptotic neurons in the hippocampus (10). Therefore, early diagnosis and timely treatment are crucial for patients with $\mathrm{CH}$.

MicroRNAs (miRNAs) are highly conserved small RNAs, 20-22 nucleotides in length, which modulate protein function via directly binding to the 3'-untranslated region (3'-UTR) of their target mRNAs (11). Recent studies have demonstrated that changes in miRNA levels are involved in numerous diseases, including myocardial ischemia (12), inflammation (13), diabetes $(14)$ and $\mathrm{CH}(4,8,9)$. In addition, miRNAs serve important functions in physiological processes, such as post-transcriptional regulation, cell proliferation and apoptosis. For example, You et al (15) found that miR-498 suppressed gastric cancer cell proliferation, migration and invasion via targeting B lymphoma Mo-MLV insertion region 1 homolog and inactivating the AKT pathway. Zhou et al (16) reported that miR-429 attenuated neuroblastoma cell viability, migration and 
invasion via the nuclear factor- $\kappa \mathrm{B}$ pathway. Furthermore, it has been reported that miR-489-3p serves key functions in several diseases. For example, Chen et al (17) demonstrated that increased expression of miR-489-3p and miR-630 inhibited oxaliplatin uptake in renal cell carcinoma via targeting octamer-binding protein 2. Additionally, Kuppa et al (18) reported that autotaxin accelerated cancer development via enhancing the expression of mitogen-activated protein kinase kinase 1 and overriding the role of miR-489-3p. However, the role of miR-489-3p in neuronal cells and $\mathrm{CH}$ remains elusive. Therefore, the present study was undertaken to investigate the role of miR-489-3p in $\mathrm{CH}$ and elucidate the potential underlying mechanisms.

Translationally controlled tumor protein 1 (TPT1), a multifunctional protein, has been evidenced to be highly expressed in various diseases, including polycystic ovary syndrome (19) and breast cancer (20), and is involved in cell proliferation, invasion, cell cycle progression and apoptosis (21). Furthermore, accumulating evidence has suggested that miR-489-3p inhibits glioblastoma progression via downregulating TPT1 (22).

The aim of the present study was to investigate the effects of miR-489-3p on hippocampal neuronal cell apoptosis in $\mathrm{CH}$ in vivo and vitro and to determine the role of the TPT1/Pim-3 signaling pathway in $\mathrm{CH}$, thereby improving our understanding of the molecular biology of the $\mathrm{CH}$.

\section{Materials and methods}

Animals. A total of 50 female pregnant Sprague Dawley

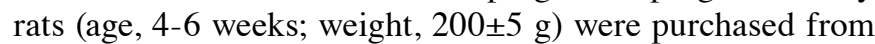
the Experimental Animal Center of Shanghai and kept in a controlled environment (temperature, $22 \pm 1^{\circ} \mathrm{C}$; humidity, 50-60\%; 12-h light/dark cycle). All animal experiments were carried out according to the guidelines provided by the National Institutes of Health (NIH) for the Care and Use of Laboratory Animals. The study protocol was approved by the Animal Ethics Committee of the Experimental Animal Center of Yancheng Maternal and Child Health Hospital.

Establishment of the $\mathrm{CH}$ model. To establish the $\mathrm{CH}$ rat model, pregnant rats were intraperitoneally injected with propylthiouracil (50 mg/day) starting on gestational day 15 and then daily thereafter until parturition, in order to generate pups with $\mathrm{CH}(4,23)$. For $\mathrm{CH}$ therapy, the newborn rats (12 days old) were anesthetized with intraperitoneal injection of $2 \%$ pentobarbital sodium (40 mg/kg), and then their skulls were opened as previously described (9). Subsequently, inhibitor control (5'-CAGUACUUUUGUGUAGUACAA-3'; GenePharma Co., Ltd.), miR-489-3p inhibitor (5'-GCUGCCGUAUAU GUGAUGUCAC-3'; GenePharma Co., Ltd.), miR-489-3p inhibitor + control-shRNA (cat. no. sc-108060; Santa Cruz Biotechnology, Inc.), or miR-489-3p inhibitor + TPT1-shRNA (cat. no. sc-40675-SH; Santa Cruz Biotechnology, Inc.) were injected into the left lateral ventricle of the 12-day-old rats using micro-syringes. The newborn rats were divided into the following six groups $(n=8)$ as follows: Control; $\mathrm{CH}$; inhibitor control; miR-489-3p inhibitor; miR-489-3p inhibitor + control-shRNA; and miR-489-3p inhibitor + TPT1-shRNA groups. At day 21 after birth, the rats (body weight $<200 \mathrm{~g}$ ) were anesthetized by intraperitoneal injection of pentobarbital sodium $(40 \mathrm{mg} / \mathrm{kg})$ and sacrificed by cervical dislocation (death was verified by cardiac and respiratory arrest). Brain hippocampal tissues from different groups were obtained following euthanasia. After anesthesia with intraperitoneal injection of pentobarbital sodium $(40 \mathrm{mg} / \mathrm{kg})$, the mother rats were also sacrificed by cervical dislocation, and death was verified by cardiac and respiratory arrest. No rats died during the experiment. The tests were terminated when the rats had lost $>15 \%$ of their body weight (body weight prior to injection).

Primary neuron cultures. After being deeply anaesthetized with $2 \%$ sevoflurane inhalation, neurons were isolated from the hippocampal tissues of 3 normal male rats (postnatal day 0 rat pups from the control group; weight, 5-6 g) and digested with trypsin for $1 \mathrm{~h}$. Subsequently, the neurons were harvested and cultivated in DMEM supplemented with 10\% FBS (both from Gibco; Thermo Fisher Scientific, Inc.) for 4-6 h. Then, neurons were cultured in neural basal medium (Thermo Fisher Scientific, Inc.) containing $2 \% \mathrm{~B} 27,100 \mathrm{U} / \mathrm{ml}$ penicillin/streptomycin and $0.5 \mathrm{mmol} / 1$ glutamine (Gibco; Thermo Fisher Scientific, Inc.) at $37^{\circ} \mathrm{C}$ in a humidified $5 \% \mathrm{CO}_{2}$ incubator.

Cell transfection. The inhibitor control (5'-CAGUACUUU UGUGUAGUACAA-3'; GenePharma Co., Ltd.), miR-489-3p inhibitor (5'-GCUGCCGUAUAUGUGAUGUCAC-3'; GenePharma Co., Ltd.), control-shRNA (cat. no. sc-108060; Santa Cruz Biotechnology, Inc.) and TPT1-shRNA (cat. no. sc-40675-SH; Santa Cruz Biotechnology, Inc.) were synthesized by Shanghai GenePharma Co., Ltd. and transfected into neurons using the Lipofectamine ${ }^{\circledR} 2000$ reagent (Invitrogen; Thermo Fisher Scientific, Inc.) according to the manufacturer's protocol. Following incubation for $48 \mathrm{~h}$, the transfection efficiency was confirmed using reverse transcription-quantitative PCR (RT-qPCR) and western blot analyses.

MTT assay. Cell proliferation was assessed using an MTT assay. Briefly, neurons $\left(5 \times 10^{4}\right.$ cells per well) were seeded into 96 -well plates in triplicate, transfected with inhibitor control, miR-489-3p inhibitor, control-shRNA or TPT1-shRNA, and cultured at $37^{\circ} \mathrm{C}$ for $48 \mathrm{~h}$. Following transfection, each well was supplemented with $20 \mu \mathrm{l}$ MTT solution (Sigma-Aldrich; Merck KGaA) and the cells were cultured for an additional $4 \mathrm{~h}$. Subsequently, the medium was discarded, and $100 \mu \mathrm{l}$ DMSO was added to dissolve the formazan. The absorbance at $490 \mathrm{~nm}$ was determined using a microplate reader (BioTek Instruments, Inc.).

Cell apoptosis assay. Treated neurons were digested, cleaned and collected at $4^{\circ} \mathrm{C}$ overnight. For the detection of cell apoptosis, the Annexin V-FITC/propidium iodide apoptosis detection kit (Beyotime Institute of Biotechnology) was used according to the manufacturer's protocol. Subsequently, apoptotic cells were identified using the FACScan flow cytometry system (BD Biosciences), and their number was measured using the FlowJo 7.6.1 software (BD Biosciences).

Dual luciferase reporter assay. TargetScan bioinformatics software (version 7.2; http://www.targetscan.org/vert_72/) was employed to identify the potential targets of miR-489-3p. Subsequently, the 3'-UTR of TPT1 containingmiR-489-3p-binding sites was sub-cloned into pMIR vectors (Ambion; Thermo Fisher 
Scientific, Inc.) to generate the TPT1 wild-type (TPT1-WT) and TPT1 mutant (TPT1-MUT) plasmids. TPT1 was identified as a possible target of miR-489-3p. A QuikChange Site-Directed Mutagenesis Kit (Stratagene; Agilent Technologies, Inc.) was applied according to the manufacturer's instructions to point-mutate the miR-489-3p-binding domain in the 3'-UTR of TPT1. For luciferase reporter activity analysis, 293T cells were co-transfected with $1 \mathrm{ng}$ TPT1-WT or $1 \mathrm{ng}$ TPT1-MUT and $100 \mathrm{nM}$ miR-489-3p mimic (5'-GUGACAUCACAUAUACGG CAGC-3'; Suzhou GenePharma Co., Ltd.) or 100 nM mimic control (5'-UUGUCCGAACGUGUCACGUTT-3'; Suzhou GenePharma Co., Ltd.) with Lipofectamine ${ }^{\circledR} 2000$ (Invitrogen; Thermo Fisher Scientific, Inc.) according to the manufacturer's instructions. After $24 \mathrm{~h}$, the luciferase activity was determined using the Dual-Luciferase Reporter Assay System (Promega Corporation) and normalized to Renilla luciferase activity.

\section{Behavioral tests}

Open-field test (OFT). This test was conducted to assess the anxiety and physical activity of experimental rats (21 days old). OFT was carried out into a $48 \times 48 \times 36 \mathrm{~cm}$ opaque apparatus partitioned into 16 equal squares by white lines, including center squares and a peripheral area. Mice were left in the center squares for $5 \mathrm{~min}$ and their activity was tracked using a digital camcorder (Ethovision 2.0; Noldus Information Technology). In this test, the following four parameters were analyzed: Ambulation distance, referring to the total distance of the grid lines crossed; center square entries, referring to the frequency of squares crossed with all four paws; center area duration, referring to the accumulated time of rat in central square; and rearing, referring to the frequency of each rat standing on the hind paws. The maze was wiped with $75 \%$ ethanol prior to testing another rat.

Forced swimming test (FST). This test was conducted to assess depression-like behavior in rats (21 days old). Briefly, the rats were placed into a transparent cylinder container (diameter, $22 \mathrm{~cm}$; depth, $40 \mathrm{~cm}$ ) filled with water to a height of $30 \mathrm{~cm}$ at $25^{\circ} \mathrm{C}$ for $6 \mathrm{~min}$. Subsequently, the swimming duration of each rat was recorded. During this experiment, immobility time was defined as the time during which the rats were floating motionless or keeping their head above water for $4 \mathrm{~min}$. Swimming activity was indicated as a non-depressive behavior.

RT-qPCR analysis. Total RNA from cultured neurons or hippocampal tissues was harvested using TRIzol ${ }^{\circledR}$ reagent (Takara Biotechnology Co., Ltd.) according to the manufacturer's instructions. Subsequently, RNA was reverse-transcribed into cDNA using the cDNA Reverse Transcription kit (Takara Biotechnology Co., Ltd.) according to the manufacturer's protocol. The miR-489-3p and TPT1 expression levels were determined using the SYBR Prime Script RT-PCR Kit (Takara Biotechnology Co., Ltd.). GAPDH and U6 served as the internal controls for mRNA and miRNA expression, respectively. The primer sequences used were as follows: GAPDH, forward, 5'-ACGGATTTGGTCGTATTGG-3' and reverse, 5'-TCCCGTTCTCAGCCTTG-3'; U6, forward, 5'-CCAAGC ATCCATGTCTCAA-3' and reverse, 5'-TCCAGATTAACC CCATCC-3'; TPT1, forward, 5'-ATGATTATCTACCGGGAC CTC-3' and reverse, 5'-TACATTTTTCCATTTCTAAACCAT
CC-3'; and miR-489-3p, forward 5'-GTGACATCACATATA CGG-3' and reverse 5'-GAACATGTCTGCGTATCTC-3'. Gene expression were analyzed using the $2^{-\Delta \Delta \mathrm{Cq}}$ method (24).

Western blot analysis. Total proteins were extracted from neurons and hippocampi using a lysis buffer (Beyotime Institute of Biotechnology), and protein concentration was measured using a BCA assay (Solarbio Science and Technology Co., Ltd.). Subsequently, the protein samples were resolved on $10 \%$ SDS-PAGE and transferred onto PVDF membranes. The membranes were blocked with 5\% skimmed milk in TBS containing $0.1 \%$ Tween at room temperature for $1.5 \mathrm{~h}$. The membranes were then incubated with primary antibodies against TPT1 (cat. no. 5128; dilution, 1:1,000; Cell Signaling Technology, Inc.), Pim-3 (cat. no. 4165; dilution, 1:1,000; Cell Signaling Technology, Inc.), p-Bad (Ser112) (cat. no. 5284; dilution, 1:1,000; Cell Signaling Technology, Inc.), Bad (cat. no. 9292; dilution, 1:1,000; Cell Signaling Technology, Inc.), Bcl-xL (cat. no. 2764; dilution, 1:1,000; Cell Signaling Technology, Inc.) and GAPDH (cat. no. 5174; dilution, 1:1,000; Cell Signaling Technology, Inc.) at $4^{\circ} \mathrm{C}$ overnight. After washing with PBST, the membranes were incubated with corresponding secondary antibody (cat. no. 7074; dilution, 1:1,000; Cell Signaling Technology, Inc.) for $2 \mathrm{~h}$ at room temperature. Finally, the protein bands were quantified using an ECL reagent (Cytiva) according to the manufacturer's instructions.

Statistical analysis. Data are expressed as the mean \pm standard deviation from three independent experiments. GraphPad Prism 6 (GraphPad Software, Inc.) and SPSS 21.0 software (IBM Corp.) were employed for statistical analyses. The variables were analyzed using Student's t-test or one-way ANOVA followed by Tukey's post hoc test. $\mathrm{P}<0.05$ was considered to indicate a statistically significant difference.

\section{Results}

miR-489-3p is upregulated in hippocampal tissues of $\mathrm{CH}$ rats via targeting TPT1. The expression of miR-489-3p in the hippocampal tissue of $\mathrm{CH}$ rats was determined by RT-qPCR analysis. The results revealed that the miR-489-3p expression levels were higher in the hippocampal tissues of $\mathrm{CH}$ rats compared with those in the control group (Fig. 1A). To verify the mechanisms underlying the effects of miR-489-3p, bioinformatics analysis was performed to predict its target genes. The analysis identified a potential binding site of miR-489-3p on TPT1 3'-UTR (Fig. 1B). Previous reports have indicated that TPT1 is a target gene of miR-489-3p. Therefore, a dual luciferase reporter assay was performed to verify the association between miR-489-3p and TPT1. It was first confirmed that, compared with the mimic control group, miR-489-3p mimic significantly enhanced miR-489-3p expression in $293 \mathrm{~T}$ cells (Fig. 1C). As shown in Fig. 1D, miR-489-3p notably decreased luciferase activity in the TPT1-WT group, but not in the TPT1-MUT group, compared with the control group. These findings suggested that miR-489-3p directly targets TPT1. Furthermore, RT-qPCR and western blot assays were performed to assess whether miR-489-3p regulates TPT1 expression. RT-qPCR analysis indicated that TPT1 mRNA was 

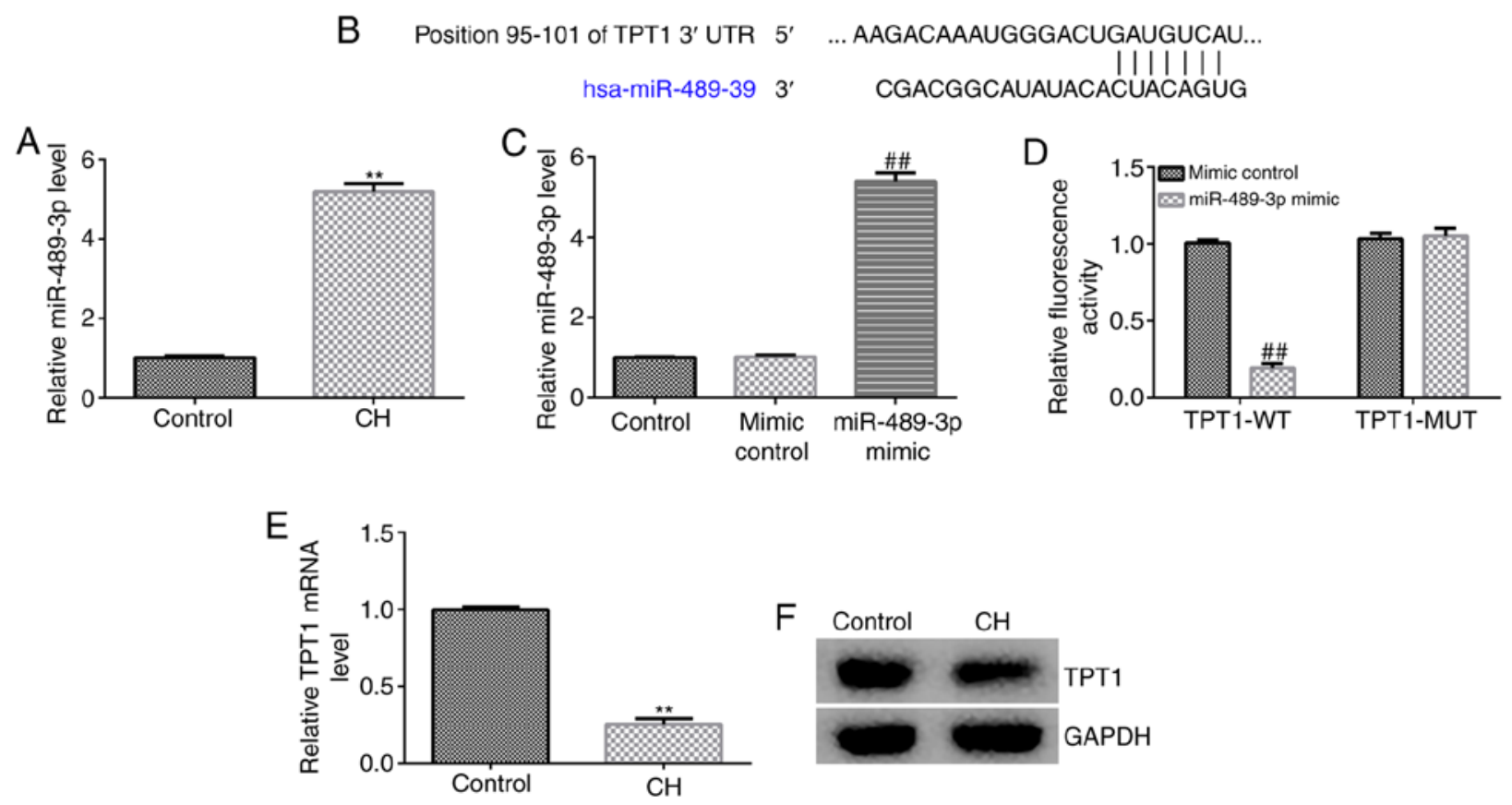

Figure 1. miR-489-3p is overexpressed in $\mathrm{CH}$ rats and acts via targeting TPT1. Pregnant rats were injected with propylthiouracil to induce $\mathrm{CH}$ in rat pups. (A) RT-qPCR analysis was performed to examine the expression levels of miR-489-3p in the hippocampal tissues of rats with or without $\mathrm{CH}$. (B) Bioinformatics analysis predicted a miR-489-3p-binding site on the TPT1 3'-UTR. (C) RT-qPCR analysis was performed to examine the expression levels of miR-489-3p in 293T cells transfected with mimic control or miR-489-3p mimic for $24 \mathrm{~h}$. (D) Dual luciferase reporter assay was performed to verify the potential association between miR-489-3p and TPT1. (E) RT-qPCR analysis was applied to measure TPT1 mRNA expression levels. (F) TPT1 protein expression levels in the hippocampal tissues of rats with or without $\mathrm{CH}$ were detected using western blot assay. ${ }^{* *} \mathrm{P}<0.01$ vs. the control group; ${ }^{\# \#} \mathrm{P}<0.01$ vs. the mimic control group. miR, microRNA; CH, congenital hypothyroidism; TPT1, translationally controlled tumor protein 1; 3'-UTR, 3'-untranslated region; RT-qPCR, reverse transcription-quantitative PCR; WT, wild-type; MUT, mutant.

notably reduced in $\mathrm{CH}$ rat hippocampal tissues compared with the control group (Fig. 1E). Similar findings were observed using western blot assay (Fig. 1F), as the TPT1 protein was found to be markedly downregulated in the hippocampal tissues of $\mathrm{CH}$ rats. These results indicated that miR-489-3p may be involved in $\mathrm{CH}$ progression via negatively regulating TPT1 expression.

miR-489-3p inhibitor improves $\mathrm{CH}$ rat behavior and reduces $\mathrm{CH}$-mediated neuronal cell apoptosis. To determine whether miR-489-3p is involved in $\mathrm{CH}$, inhibitor control, miR-489-3p inhibitor, miR-489-3p inhibitor + control-shRNA or miR-489-3p inhibitor + TPT1-shRNA were injected into rats to establish $\mathrm{CH}$ rat models. The rats were divided into the following six groups: Control; $\mathrm{CH}$; inhibitor control; miR-489-3p inhibitor; miR-489-3p inhibitor + control-shRNA; and miR-489-3p inhibitor + TPT1-shRNA groups. First, RT-qPCR analysis was performed to determine miR-489-3p and TPT1 expression in rat hippocampal tissues in different groups. Compared with the control group, miR-489-3p was upregulated in the model group, while this increase was reversed following treatment with miR-489-3p inhibitor (Fig. 2A). Furthermore, the expression of TPT1 was lower in the model group compared with that in the control group. In addition, compared to the $\mathrm{CH}+$ inhibitor control group, TPT1 was upregulated in $\mathrm{CH}$ rats in the $\mathrm{CH}+$ miR-489-3p inhibitor group, and this effect was significantly reversed by TPT1-shRNA (Fig. 2B).

Subsequently, behavioral tests were performed to evaluate the neuronal injury-induced behavioral variation following treatment with miR-489-3p inhibitor. miR-489-3p inhibitor attenuated rat anxiety- and depressive-like behavior, as observed in the FST (Fig. 2C) and OFT (Fig. 2D and E), respectively. These results indicated that miR-489-3p inhibitor significantly improved the behavior of $\mathrm{CH}$ rats. Furthermore, cell apoptosis assay was performed to assess the effect of miR-489-3p inhibitor on neuronal cell apoptosis. The data demonstrated that miR-489-3p inhibitor suppressed $\mathrm{CH}$-induced neuronal cell apoptosis, while this effect was reversed by TPT1-shRNA (Fig. 2F and G). The aforementioned findings suggested that miR-489-3p inhibitor may relieve $\mathrm{CH}$ via regulating neuronal cell apoptosis.

TPT1-shRNA reverses the effects of miR-489-3p inhibitor on TPT1 expression in neuronal cells. Subsequently, the effect of miR-489-3p inhibitor on TPT1 expression was explored in neuronal cells by knockdown assay. miR-489-3p inhibitor, inhibitor control, miR-489-3p inhibitor + control-shRNA or miR-489-3p inhibitor + TPT1-shRNA were transfected into neuronal cells, and transfection efficiency was determined using RT-qPCR and western blot assays. The RT-qPCR results suggested that miR-489-3p inhibitor successfully downregulated miR-489-3p expression in neuronal cells compared with the inhibitor control group (Fig. 3A). Furthermore, in neuronal cells, transfection with TPT1-shRNA downregulated TPT1 expression at both the mRNA and protein levels compared with the control-shRNA group (Fig. 3B and C). In addition, compared with the inhibitor control group, miR-489-3p inhibitor upregulated TPT1 mRNA and protein expression levels. On the contrary, the effects of miR-489-3p inhibitor were reversed following transfection of neuronal cells with 

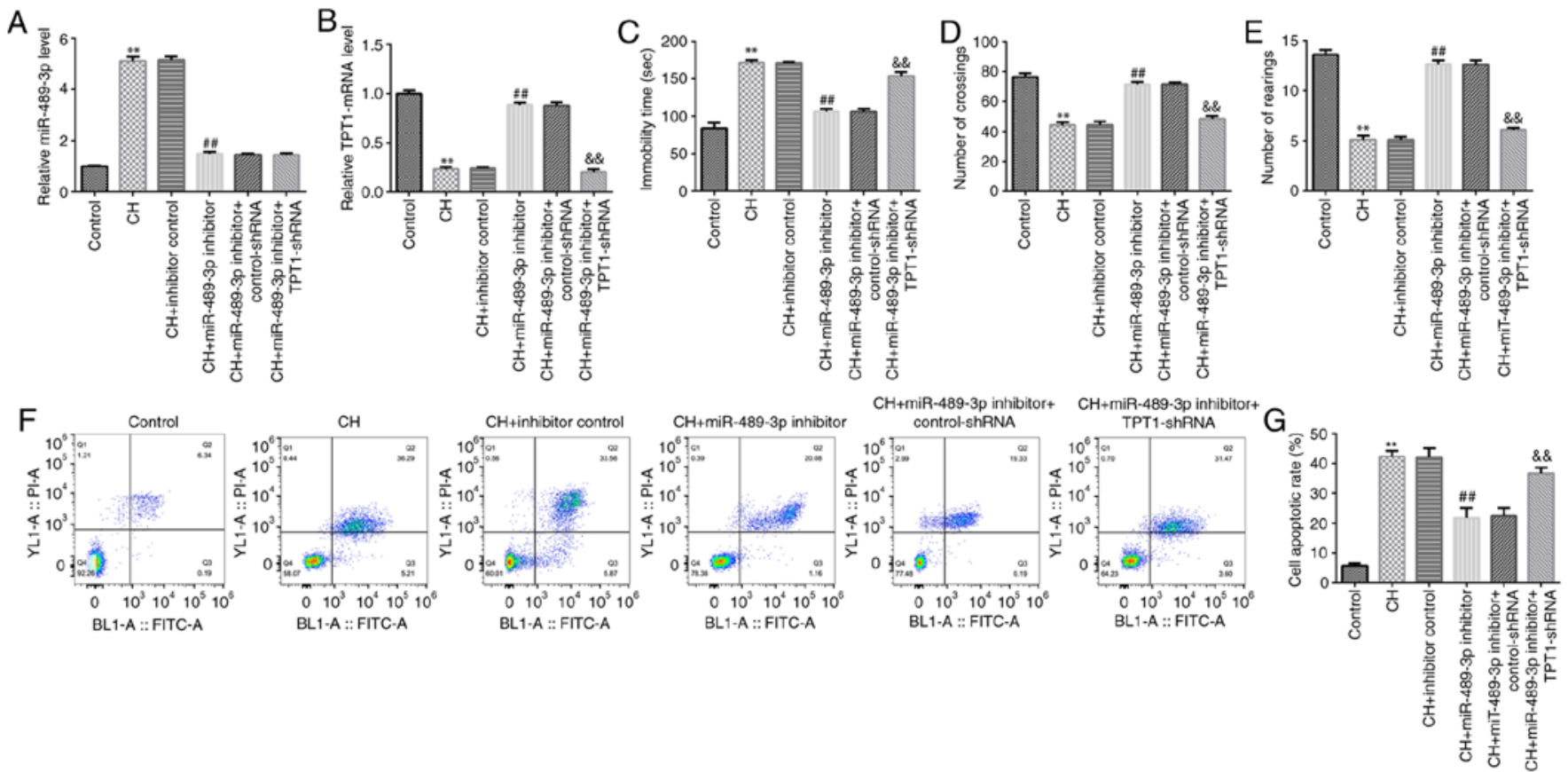

Figure 2. Effect of miR-489-3p inhibitor and TPT1-shRNA on CH rats. Inhibitor control, miR-489-3p inhibitor, miR-489-3p inhibitor + control-shRNA or miR-489-3p inhibitor + TPT1-shRNA were injected into CH rats. (A) The miR-489-3p expression levels were determined by RT-qPCR analysis. (B) RT-qPCR analysis of TPT1 expression in the six groups. The effects of miR-489-3p inhibitor on (C) depressive- and (D and E) anxiety-like behaviors were determined using forced swimming and open-field tests, respectively. (F) Rat neuronal cell apoptosis was determined by flow cytometry in different groups. (G) Quantified cell apoptosis rate. ${ }^{* *} \mathrm{P}<0.01$ vs. the control group; ${ }^{\# \#} \mathrm{P}<0.01$ vs. the $\mathrm{CH}+$ inhibitor control group; ${ }^{\text {\& }} \mathrm{P}<0.01$ vs. the $\mathrm{CH}+$ miR-489-3p inhibitor + control-shRNA group. miR, microRNA; TPT1, translationally controlled tumor protein 1; CH, congenital hypothyroidism; RT-qPCR, reverse transcription-quantitative PCR.

TPT1-shRNA (Fig. 3D and E). Taken together, these results confirmed that miR-489-3p negatively regulated TPT1 expression in neuronal cells.

TPT1 silencing abolishes the effect of miR-489-3p inhibitor on neuronal cell viability and apoptosis via the TPT1/Pim-3 signaling pathway. To further explore the effect of miR-489-3p on $\mathrm{CH}, \mathrm{MTT}$ and flow cytometry assays were conducted to assess neuronal cell viability and apoptosis, respectively. Neuronal cells were transfected with miR-489-3p inhibitor, inhibitor control, miR-489-3p inhibitor + control-shRNA or miR-489-3p inhibitor + TPT1-shRNA. As shown in Fig. 4A, miR-489-3p inhibitor markedly increased cell viability compared with that observed in the inhibitor control group. However, TPT1-shRNA reversed this effect and significantly inhibited cell viability (Fig. 4A). In addition, flow cytometry revealed that miR-489-3p inhibitor significantly reduced cell apoptosis, while TPT1-shRNA rescued the inhibitory effects of miR-489-3p inhibitor on neuronal cells (Fig. 4B).

Additionally, the mechanism underlying the effect of the miR-489-3p inhibitor on the suppression of neuronal cell apoptosis was investigated. The expression levels of the apoptosis-related proteins Pim-3, Bcl-xL p-Bad (Ser112) and Bad were detected using western blot assay. The results demonstrated that downregulation of miR-489-3p increased the expression levels of Pim-3, Bcl-xL, p-Bad (Ser112) and the ratio of p-Bad (Ser112)/Bad in neuronal cells. However, this effect was reversed following co-transfection with TPT1-shRNA (Fig. 4C-F). These findings suggested that miR-489-3p may promote $\mathrm{CH}$ progression via mediating neuronal cell apoptosis by targeting TPT1.

\section{Discussion}

$\mathrm{CH}$, one of the most common preventable diseases, may be associated with mental retardation, various developmental barriers and high anxiety scores (25). Alcigir et al (5) revealed the neuroprotective activity of cannabinoid receptor 2 against oxidative stress and apoptosis in rat pups with experimentally induced $\mathrm{CH}$. Li et al (9) reported that miR-124-3p attenuated the progression of $\mathrm{CH}$ via targeting programmed cell death protein 6. miRNAs are small non-coding molecules that play key roles in myocardial diseases (26) and diseases of the nervous system, such as Alzheimer's (27) and Parkinson's disease (28). In addition, miRNAs play critical roles in the development of tumors, thus serving as diagnostic markers (29). Several studies have evidenced that numerous miRNAs are differentially expressed in $\mathrm{CH}$ tissues and may be involved in the development of $\mathrm{CH}(4,8,9)$. However, the expression and associated mechanisms of action of miR-489-3p in $\mathrm{CH}$ remain elusive. Therefore, the present study was undertaken to investigate the exact role of miR-489-3p in $\mathrm{CH}$.

Propylthiouracil was injected into pregnant rats to establish a CH model, and the results revealed that miR-489-3p was markedly upregulated in the hippocampi of $\mathrm{CH}$ rats compared with the control group. Furthermore, the potential targets of miR-489-3p were predicted using bioinformatics assay. Therefore, a binding site for miR-489-3p was identified in the TPT1 3'-UTR. The association between miR-489-3p and TPT1 3'-UTR was confirmed by a dual luciferase reporter assay. TPT1, a highly conserved protein, has been reported to be combined with other proteins to alter protein expression levels (30). Our findings were consistent with those of 


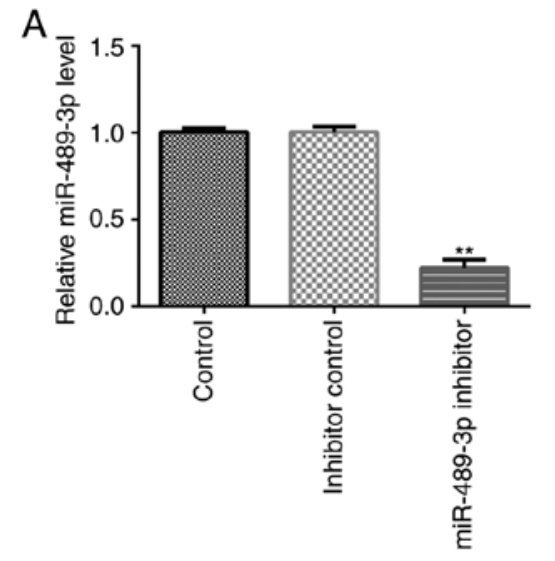

$\mathrm{B}$

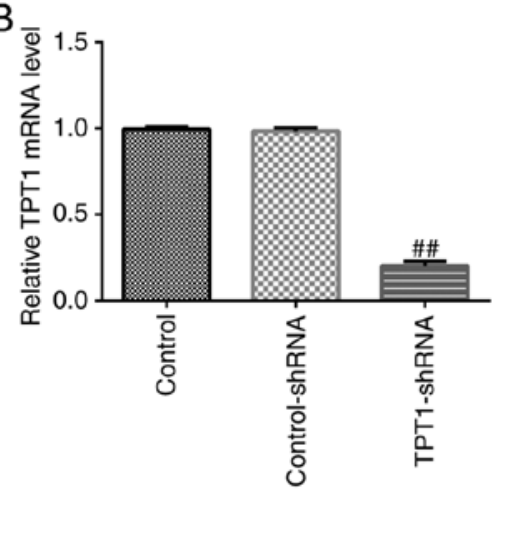

C

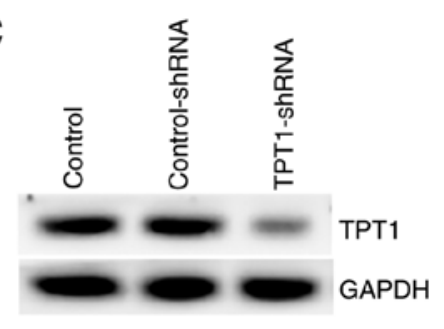

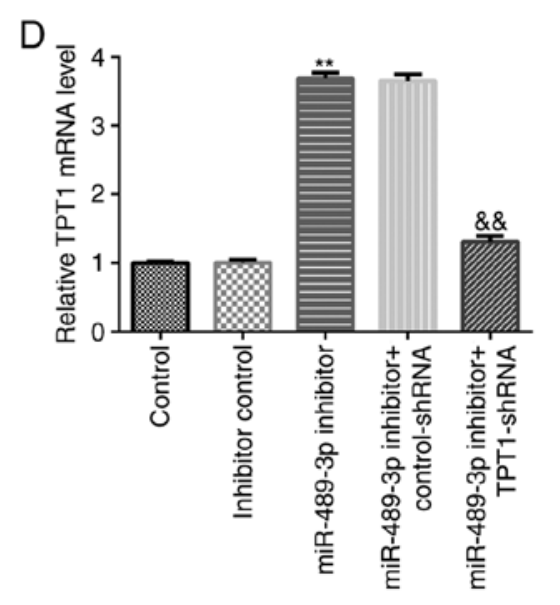

$\mathrm{E}$

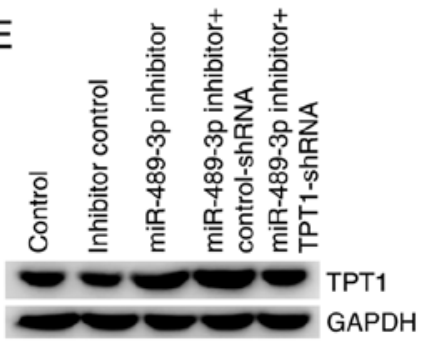

Figure 3. Effect of miR-489-3p inhibitor and TPT1-shRNA on TPT1 expression in cultured neuronal cells. miR-489-3p inhibitor, inhibitor control, miR-489-3p inhibitor + control-shRNA or miR-489-3p inhibitor + TPT1-shRNA were transfected into neuronal cells. (A) The levels of miR-489-3p were measured using RT-qPCR analysis in the inhibitor control and miR-489-3p inhibitor groups. (B) RT-qPCR and (C) western blot analysis of TPT1 expression in neuronal cells following treatment with control-shRNA and TPT1-shRNA. (D) RT-qPCR and (E) western blot assays were conducted to assess TPT1 expression in neuronal cells following transfection with miR-489-3p inhibitor + control-shRNA or miR-489-3p inhibitor + TPT1-shRNA. ${ }^{* *}$ P $<0.01$ vs. the inhibitor control group; ${ }^{\#} \mathrm{P}<0.01$ vs. the control-shRNA group; ${ }^{\&} \& \mathrm{P}<0.01$ vs. the miR-489-3p inhibitor + control-shRNA group. miR, microRNA; TPT1, translationally controlled tumor protein 1; RT-qPCR, reverse transcription-quantitative PCR.

previous reports suggesting that TPT1 was directly targeted by miR-489-3p (22). The present study demonstrated that TPT1 was downregulated in the hippocampal tissues of $\mathrm{CH}$ rats compared with the control group. The aforementioned observations indicated that miR-489-3p may be involved in $\mathrm{CH}$ via negatively regulating TPT1 expression.

Dysregulation of miRNA expression has been associated with several diseases. To identify whether miR-489-3p affects the progression of $\mathrm{CH}$, inhibitor control, miR-489-3p inhibitor, miR-489-3p inhibitor + control-shRNA or miR-489-3p inhibitor + TPT1-shRNA were injected into rats to establish $\mathrm{CH}$ models. As dysregulation of miRNAs may alter behavioral cognition (31), the present study further evaluated the effects of miR-489-3p inhibitor and TPT1-shRNA on rat behavior. Treatment with miR-489-3p inhibitor led to spatial memory reconstruction, and decreased anxiety- and depressive-like behaviors. It has been previously demonstrated that several miRNAs may protect neurons against apoptosis in various diseases (32). The results of the present study suggested that $\mathrm{CH}$ may promote neuronal apoptosis, whereas transfection with miR-489-3p inhibitor attenuated this effect, which was eliminated following treatment with TPT1-shRNA. These results indicated that miR-489-3p inhibitor may relieve $\mathrm{CH}$ via regulating neuronal cell apoptosis.
The effects of miR-489 on cell viability and apoptosis have been previously reported. Wu et al (33) demonstrated that miR-489 suppressed multiple myeloma cell proliferation via inhibiting lactate dehydrogenase A-mediated aerobic glycolysis. Consistent with this finding, Gao et al (34) reported that miR-489 may suppress tumor growth and invasion via targeting histone deacetylase 7 in colorectal cancer. In addition, it has been demonstrated that TPT1 promotes cell proliferation and attenuates apoptosis (21). Hippocampal neuronal cell apoptosis is considered as the key characteristic of $\mathrm{CH}$ (35). Therefore, the effect of miR-489-3p on hippocampal neuronal cell apoptosis was explored. The in vitro results indicated that miR-489-3p inhibitor enhanced neuronal cell viability and inhibited apoptosis, which was alleviated by TPT1-shRNA. It has been reported that Pim-3, a member of the proto-oncogene Pim family, regulates cell viability during cancer development (36). For example, Fan et al (37) revealed that Pim-3 reduced cell proliferation and apoptosis in A549 lung adenocarcinoma cells. Additionally, Pim-3 enhanced melanoma cell migration and invasion via promoting signal transducer and activator of transcription 3 phosphorylation (38). Therefore, the expression of apoptosis-related proteins was further assessed using western blot analysis. The data demonstrated 

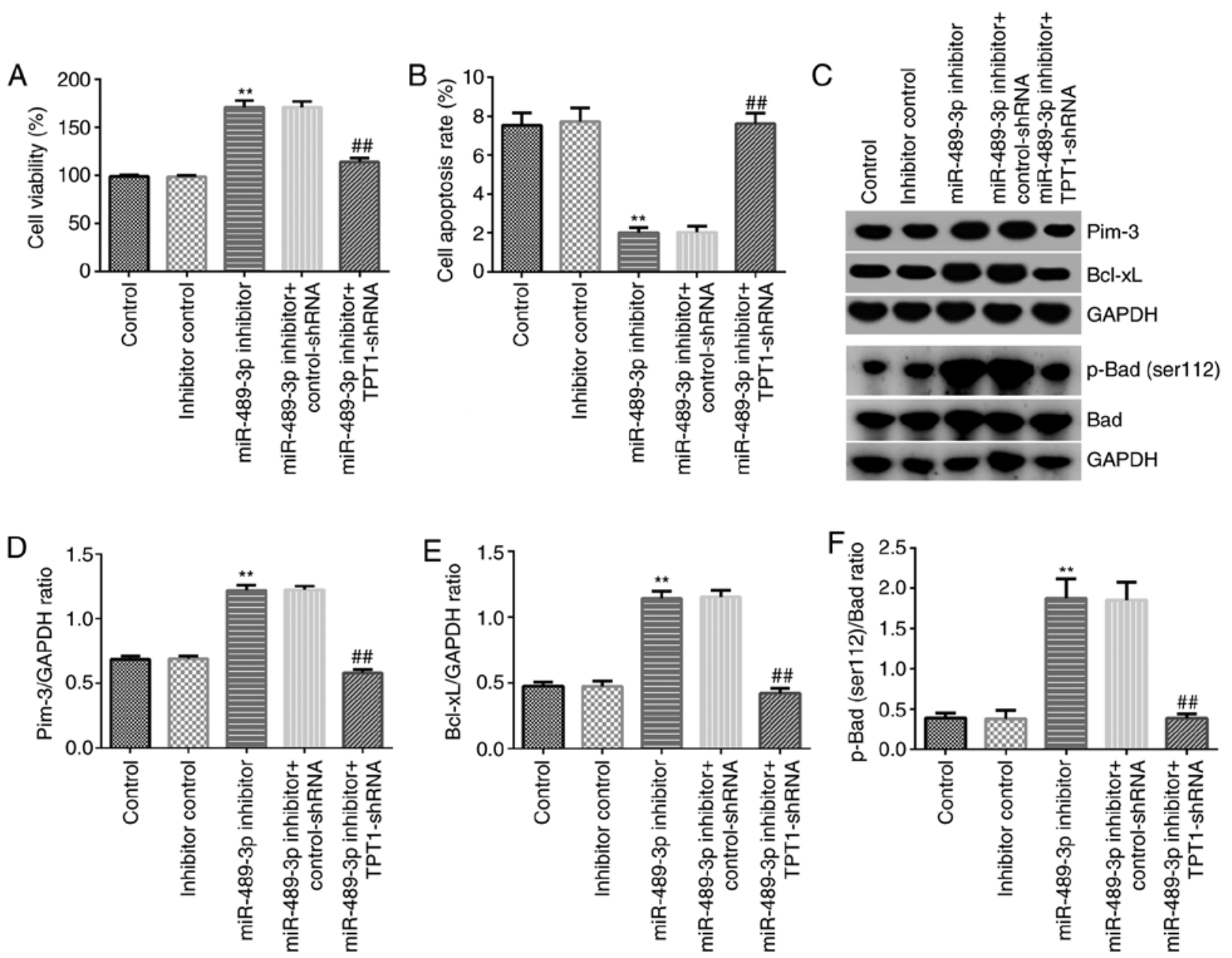

Figure 4. TPT1-shRNA reversed the effects of miR-489-3p inhibitor on neuronal cell viability and apoptosis via the TPT1/Pim-3 pathway. miR-489-3p inhibitor, inhibitor control, miR-489-3p inhibitor + control-shRNA or miR-489-3p inhibitor + TPT1-shRNA were transfected into neuronal cells. (A) Neuronal cell viability was assessed using an MTT assay. (B) Neuronal cell apoptosis was measured using flow cytometry. (C) The Pim-3, Bcl-xL, p-Bad (Ser112) and Bad protein expression levels in the six different groups were determined using western blot assay. (D-F) The Pim-3/GAPDH, Bcl-xL/GAPDH and p-Bad (Ser112)/Bad ratios were calculated. ${ }^{* *} \mathrm{P}<0.01$ vs. the inhibitor control group; ${ }^{\# \#} \mathrm{P}<0.01$ vs. the control-shRNA group. miR, microRNA; TPT1, translationally controlled tumor protein 1 .

that the expression levels of Pim-3, p-Bad (Ser112) and Bcl-xL were significantly increased by miR-489-3p inhibitor in hippocampal neurons. Furthermore, transfection with TPT1-shRNA abolished the miR-489-3p inhibitor-mediated upregulation of Pim-3, p-Bad (Ser112) and Bcl-xL.

A limitation of the present study was that it did not include a group treated exclusively with sh-TPT1, which would further improve our understanding of the regulatory association between miR-489-3p and TPT1.

In conclusion, the results of the present study suggested that the miR-489-3p inhibitor may relieve $\mathrm{CH}$-associated neurological damage via regulating the TPT1/Pim-3 signaling pathway. The findings of the study may contribute to the better understanding of the molecular biology of $\mathrm{CH}$.

\section{Acknowledgements}

Not applicable.

\section{Funding}

No funding was received.

\section{Availability of data and materials}

The datasets used and/or analyzed during the current study are available from the corresponding author on reasonable request.

\section{Authors' contributions}

QL and YL designed the current study, collected the data, performed statistical analysis and interpretation, and prepared the manuscript. YZ collected and analyzed the data. All authors read and approved the final manuscript. QL and YZ confirm the authenticity of all the raw data.

\section{Ethics approval and consent to participate}

All animal experiments were carried out according to the guidelines provided by the National Institutes of Health (NIH) for the Care and Use of Laboratory Animals. The study protocol was approved by the Animal Ethics Committee of the Experimental Animal Center of Yancheng Maternal and Child Health Hospital. 


\section{Patient consent for publication}

Not applicable.

\section{Competing interests}

The authors declare that they have no competing interests.

\section{References}

1. Rousseau JP, Buteau-Poulin A and Kinkead R: Maternal thyroid hormone deficiency and cardiorespiratory disorder in rat pups. Exp Neurol 320: 112960, 2019.

2. Tiosano D, Pannain S, Vassart G, Parma J, Gershoni-Baruch R, Mandel H, Lotan R, Zaharan Y, Pery M, Weiss R, et al: The hypothyroidism in an inbred kindred with congenital thyroid hormone and glucocorticoid deficiency is due to a mutation producing a truncated thyrotropin receptor. Thyroid 9: 887-894, 1999.

3. Zhang L, Blomgren K, Kuhn HG and Cooper-Kuhn CM: Effects of postnatal thyroid hormone deficiency on neurogenesis in the juvenile and adult rat. Neurobiol Dis 34: 366-374, 2009.

4. Meng T, Shen S, Li C and Liu X: MicroRNA-1236-3p/ translationally controlled tumor protein (TPT1) axis participates in congenital hypothyroidism progression by regulating neuronal apoptosis. Exp Ther Med 19: 459-466, 2020.

5. Alcigir ME, Dogan HO, Atalay VS and Yilmaz FM: Neuroprotective activity of cannabinoid receptor-2 against oxidative stress and apoptosis in rat pups having experimentally-induced congenital hypothyroidism. Dev Neurobiol 77: 1334-1347, 2017.

6. Akin MA, Aydogan S, Gunes T, Artis AS, Karakukcu M and Kurtoglu S: Changes of red blood cell rheology in newborns with congenital hypothyroidism during treatment. J Matern Fetal Neonatal Med 26: 1532-1536, 2013.

7. Vetrovoy O, Sarieva K, Lomert E, Nimiritsky P, Eschenko N, Galkina O, Lyanguzov A, Tyulkova E and Rybnikova E: Pharmacological HIF1 inhibition eliminates down-regulation of the pentose phosphate pathway and prevents neuronal apoptosis in rat hippocampus caused by severe hypoxia. J Mol Neurosci 70: 635-646, 2020.

8. Shao Q, Jiang W and Jin Y: MiR-124 effect in neurons apoptosis in newborn rat with thyroid hypofunction. Int J Clin Exp Pathol 8: 14465-14471, 2015.

9. Li W, Song D, Sun Y, Lv Y and Lv J: microRNA-124-3p inhibits the progression of congenital hypothyroidism via targeting programmed cell death protein 6. Exp Ther Med 15: 5001-5006, 2018.

10. Huang XW, Yin HM, Ji C, Qin YF, Yang RW and Zhao ZY: Effects of perinatal hypothyroidism on rat behavior and its relation with apoptosis of hippocampus neurons. J Endocrinol Invest 31: 8-15,2008.

11. Schira-Heinen J, Czapla A, Hendricks M, Kloetgen A, Wruck W, Adjaye J, Kögler G, Werner Müller H, Stühler K and Trompeter HI: Functional omics analyses reveal only minor effects of microRNAs on human somatic stem cell differentiation. Sci Rep 10: 3284, 2020.

12. Omidkhoda N, Wallace HA, Reiter RJ and Karimi G: The role of microRNAs on endoplasmic reticulum stress in myocardial ischemia and cardiac hypertrophy. Pharmacol Res 150: 104516, 2019.

13. Wu S, Wang J, Li J and Li F: microRNA-21 aggravates lipopolysaccharide-induced inflammation in MH7A cells through targeting SNF5. Inflammation 43: 441-454, 2020.

14. Sohrabifar N, Ghaderian S, Vakili H, Ghaedi H, Rouhani B, Jafari $\mathrm{H}$ and Heidari L: MicroRNA-copy number variations in coronary artery disease patients with or without type 2 diabetes mellitus. Arch Physiol Biochem: 1-7, 2019.

15. You D, Wang D, Liu P, Chu Y, Zhang X, Ding X, Li X, Mao T, Jing X, Tian Z and Pan Y: MicroRNA-498 inhibits the proliferation, migration and invasion of gastric cancer through targeting BMIand suppressing AKT pathway. Hum Cell 33: 366-376, 2020

16. Zhou X, Lu H, Li F, Hao X, Han L, Dong Q and Chen X: MicroRNA-429 inhibits neuroblastoma cell proliferation, migration and invasion via the NF- $\mathrm{kB}$ pathway. Cell Mol Biol Lett 25 $5,2020$.

17. Chen L, Chen L, Qin Z, Lei J, Ye S, Zeng K, Wang H, Ying M, Gao J, Zeng S and Yu L: Up-regulation of miR-489-3p and miR-630 inhibits oxaliplatin uptake in renal cell carcinoma by targeting OCT2. Acta Pharm Sin B 9: 1008-1020, 2019.
18. Kuppa SS, Jia W, Liu S, Nguyen H, Smyth SS, Mills GB, Dobbin KK, Hardman WJ and Murph MM: Autotaxin exacerbates tumor progression by enhancing MEK1 and overriding the function of miR-489-3p. Cancer Lett 432: 84-92, 2018

19. Sun X, Su S, Zhang G, Zhang H and Yu X: MiR-204 suppresses cell proliferation and promotes apoptosis in ovarian granulosa cells via targeting TPT1 in polycystic ovary syndrome. Biochem Cell Biol 97: 554-562, 2019.

20. Neuhäuser K, Küper L, Christiansen $H$ and Bogdanova $N$ : Assessment of the role of translationally controlled tumor protein 1 (TPT1/TCTP) in breast cancer susceptibility and ATM signaling. Clin Transl Radiat Oncol 15: 99-107, 2019.

21. Li R, Zhu H, Yang D, Xia J and Zheng Z: Long noncoding RNA lncBRM promotes proliferation and invasion of colorectal cancer by sponging miR-204-3p and upregulating TPT1. Biochem Biophys Res Commun 508: 1259-1263, 2019.

22. Zhang L, Wang Q, Wang F, Zhang X, Zhang L, Tang Y and Wang S: LncRNA LINC01446 promotes glioblastoma progression by modulating miR-489-3p/TPT1 axis. Biochem Biophys Res Commun 503: 1484-1490, 2018.

23. Fabian ID, Rosner M, Fabian I, Vishnevskia-Dai V, Zloto O, Shinderman Maman E, Cohen K, Ellis M,Lin HY,Hercbergs A, et al: Low thyroid hormone levels improve survival in murine model for ocular melanoma. Oncotarget 6: 11038-11046, 2015.

24. Livak KJ and Schmittgen TD: Analysis of relative gene expression data using real-time quantitative PCR and the 2(-Delta Delta C(T)) method. Methods 25: 402-408, 2001

25. Sidibé el H: Reflections on mental retardation and congenital hypothyroidism: Effects of trace mineral deficiencies. Sante 17: 41-50, 2007 (In French).

26. Boen J, Gevaert AB, De Keulenaer GW, Van Craenenbroeck EM and Segers V: The role of endothelial miRNAs in myocardial biology and disease. J Mol Cell Cardiol 138: 75-87, 2020.

27. Segaran RC, Chan LY, Wang H, Sethi G and Tang FR: Neuronal development-related miRNAs as biomarkers for Alzheimer's disease, Depression, Schizophrenia and Ionizing Radiation Exposure. Curr Med Chem: Jan 21, 2020 (Epub ahead of print) doi: 10.2174/092986732766620012112291.

28. Sadlon A, Takousis P, Alexopoulos P, Evangelou E, Prokopenko I and Perneczky R: miRNAs identify shared pathways in Alzheimer's and Parkinson's diseases. Trends Mol Med 25: 662-672, 2019.

29. Condrat CE, Thompson DC, Barbu MG, Bugnar OL, Boboc A, Cretoiu D, Suciu N, Cretoiu SM and Voinea SC: miRNAs as biomarkers in disease: Latest findings regarding their role in diagnosis and prognosis. Cells 9: 276, 2020.

30. Jian M, Du Q, Zhu D, Mao Z, Wang X, Feng Y, Xiao Z, Wang H and Zhu Y: Tumor suppressor miR-145-5p sensitizes prolactinoma to bromocriptine by downregulating TPT1. J Endocrinol Invest 42: 639-652, 2019.

31. Dong J, Liu Y,Zhan Z and Wang X: MicroRNA-132 is associated with the cognition improvement following voluntary exercise in SAMP8 mice. Brain Res Bull 140: 80-87, 2018.

32. Li LM, Luo FJ and Song X: MicroRNA-370-3p inhibits cell proliferation and induces chronic myelogenous leukaemia cell apoptosis by suppressing PDLIM1/Wnt/ $\beta$-catenin signalling. Neoplasma 67: 509-518, 2020.

33. Wu H, Wang X, Wu T and Yang S: miR-489 suppresses multiple myeloma cells growth through inhibition of LDHA-mediated aerobic glycolysis. Genes Genomics 42: 291-297, 2020.

34. Gao S, Liu H, Hou S, Wu L, Yang Z, Shen J, Zhou L, Zheng SS and Jiang B: MiR-489 suppresses tumor growth and invasion by targeting HDAC7 in colorectal cancer. Clin Transl Oncol 20: 703-712, 2018.

35. You Y, Tan J, Gong Y, Dai H, Chen H, Xu X, Yang A, Zhang Y and Bie P: MicroRNA-216b-5p functions as a tumor-suppressive RNA by targeting TPT1 in pancreatic cancer cells. J Cancer 8: 2854-2865, 2017.

36. Zan T, Piao L, Yang X, Gu Y and Liu B: Down-regulation of microRNA-124 prevents the development of acute liver failure through the upregulation of PIM-3. Exp Physiol 105: 108-119, 2020.

37. Fan X, Xie Y, Zhang L, Gao X, Han J, Chen Y, Yang J and Li S: Effect of Pim-3 down-regulation on proliferation and apoptosis in lung adenocarcinoma A549 cells. Ann Clin Lab Sci 49: 770-776, 2019.

38. Liu J, Qu X, Shao L, Hu Y, Yu X, Lan P, Guo Q, Han Q, Zhang J and Zhang C: Pim-3 enhances melanoma cell migration and invasion by promoting STAT3 phosphorylation. Cancer Biol Ther 19: 160-168, 2018.

This work is licensed under a Creative Commons Attribution-NonCommercial-NoDerivatives 4.0 International (CC BY-NC-ND 4.0) License. 